\title{
The Shoulder Instability: An Overview
}

\author{
Balaji Zacharia ${ }^{1, \odot}$ Arun Prakas ${ }^{1} \quad$ Subramanian Vaidyanathan ${ }^{2}$ Antony Roy ${ }^{1, \odot}$ \\ Mohammed Komalam Ayyub ${ }^{1}$
}

${ }^{1}$ Department of Orthopedics, Government Medical College, Kozhikode, Kerala, India

2Department of Orthopedics, Government Medical College, Thrissur, Kerala, India

\author{
Address for correspondence Balaji Zacharia, MBBS, Dortho, \\ MS, Department of Orthopedics, Government Medical College, \\ Kozhikode 673008, Kerala, India \\ (e-mail: balaji.zacharia@gmail.com).
}

\author{
Abstract \\ Keywords \\ - Bankart shoulder \\ lesion \\ - Hill-Sachs lesion \\ - Putti-Platt procedure \\ - remplissage \\ procedure \\ - shoulder instability
}

Instability of the shoulder joint is common but most of the time it is unrecognized by the treating surgeon. The symptoms can vary from subtle pain to shoulder dislocation. In many cases, there is no history of trauma. The shoulder joint is stabilized by both static and dynamic factors, and treatment is complex, with recurrence more common in young athletic individuals. Open and arthroscopic techniques of stabilization are available with specific indications for each of these methods. In this narrative review of shoulder instability, we describe the pathological anatomy, evaluation, natural history, classification, and treatment of shoulder instability.

\section{Introduction}

Shoulder instability is the abnormal movement of the humeral head relative to the glenoid during movements of the shoulder, producing pain or apprehension. ${ }^{1}$ It is a relatively common problem in young athletes. People engaged in contact sports have a higher incidence compared with the normal. There is a bimodal distribution for a shoulder dislocation in the second and sixth decades. Recurrence is common in patients younger than 20 years. ${ }^{2}$

\section{Normal Stabilizers of the Glenohumeral Joint and Pathological Anatomy}

The shoulder is an inherently unstable joint. The articular surface area of the humeral head is two to four times that of the glenoid. The stability of the glenohumeral joint is due to the complex interaction between the dynamic and static stabilizers. The capsule, concavity compression, and negative intracapsular pressure are the main static stabilizers. The deltoid, rotator cuff muscles, and inferior glenohumeral ligaments (IGL) are the dynamic stabilizers. ${ }^{3}$ The IGL is the primary restraint for anterior, posterior, and inferior translation of the humeral head beyond 45 degrees of abduction. ${ }^{4}$

An alteration of the normal position or movements of the scapula during coupled scapulohumeral motion is called scapular dyskinesia. It can be due to repeated injuries or overuse of the shoulder. The Scapular malpositioning, Inferior medial border prominence, Coracoid pain and malposition, and dysKinesis (SICK) shoulder is an overuse syndrome where an increased scapular inferior inclination and increased version are associated with anterior instability. Disruption of any of the stabilizing factors can lead to dislocation, subluxation, or apprehension associated with pain. ${ }^{5}$

The most common pathological lesion associated with anterior shoulder instability is the disruption of anteroinferior labrum-Bankart lesion ( - Fig. 1). ${ }^{6}$ IGL injury to a varying extent like anterior labral periosteal sleeve avulsion (ALPSA) (-Fig. 2), avulsion of the humeral attachment of the glenohumeral ligament (HAGL), and glenolabral articular disruption (GLAD) are the various alternate lesions seen in anterior shoulder instability (-Fig. 3). ${ }^{7}$ Bony lesions like indentation published online June 7, 2021
DOI https://doi.org/

10.1055/s-0041-1731113 ISSN 2455-7420
(C) 2021. Medical and Surgical Update Society.

This is an open access article published by Thieme under the terms of the Creative Commons Attribution-NonDerivative-NonCommercial-License, permitting copying and reproduction so long as the original work is given appropriate credit. Contents may not be used for commercial purposes, or adapted, remixed, transformed or built upon. (https://creativecommons.org/licenses/by-nc-nd/4.0/). Thieme Medical and Scientific Publishers Pvt. Ltd. A-12, 2nd Floor, Sector 2, Noida-201301 UP, India 


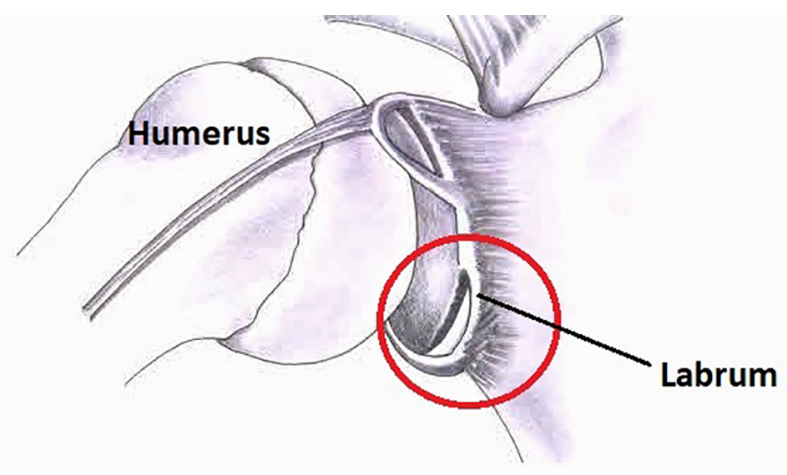

\section{Bankart Lesion}

Fig. 1 Diagrammatic representation of Bankart lesion. The anteroinferior part of the labrum detached from the glenoid. If it is associated with avulsion of fracture of the glenoid rim, the lesion is called Perthes lesion.

\section{Glenoid \\ Labral Articular Defect}

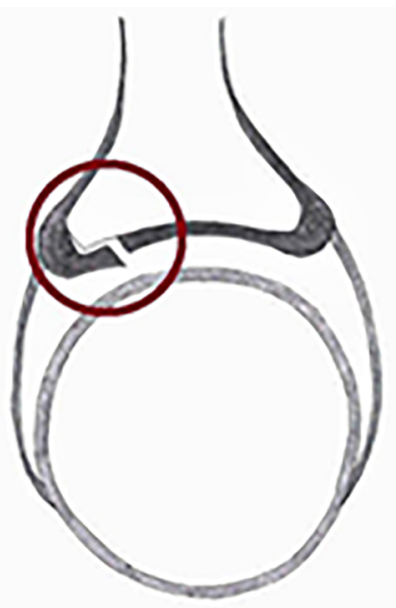

Fig. 3 Diagram showing the glenolabral articular disruption (GLAD) lesion.

fracture of humeral head-Hill-Sachs lesion and bony Bankart lesion are also seen (-Fig. 4). ${ }^{8}$

\section{Evaluation of Shoulder Instability}

A thorough physical examination is needed in all patients with shoulder instability. A detailed history including mechanism of injury, age of the first occurrence, and details of treatment taken need to be obtained. An examination to rule out generalized ligamentous laxity and any neurological disorders must be performed. ${ }^{9}$ The apprehension test, load and shift test, and Job's relocation test are useful to find out the anterior instability. Gagey's test, a hyperabduction of more than 15 degrees compared with the opposite shoulder, indicates IGL injury. ${ }^{10}$ The rotator cuff must also be evaluated. ${ }^{11}$

Standard radiographs of the shoulder is the primary form of investigation. Special views like West Point view to detect anteroinferior glenoid injury and Stryker notch view for Hill-Sachs lesion can be taken. MRI including an MR arthrogram may be useful. CT scanning helps to accurately assess the size and morphology of glenoid or humeral head defects.

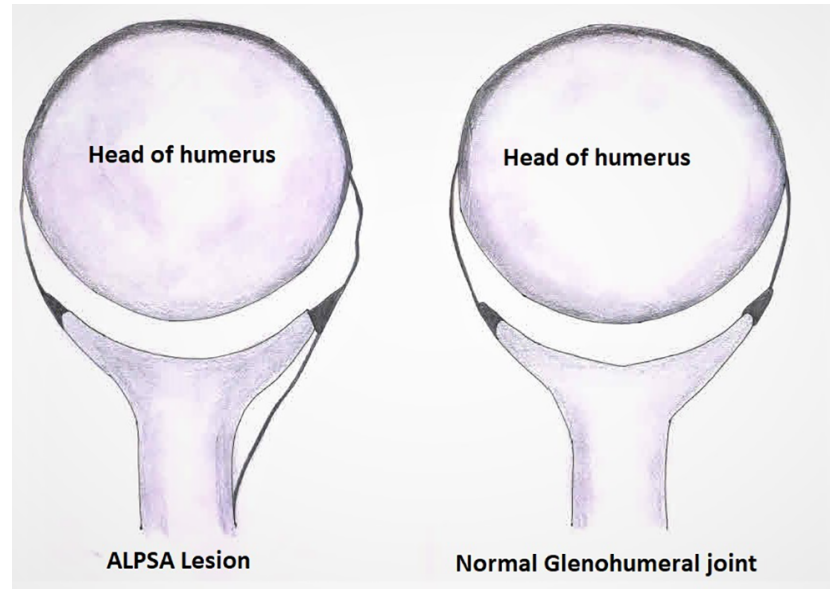

Fig. 2 Diagram showing the anterior labral periosteal sleeve avulsion (ALPSA) of the inferior glenohumeral ligament.

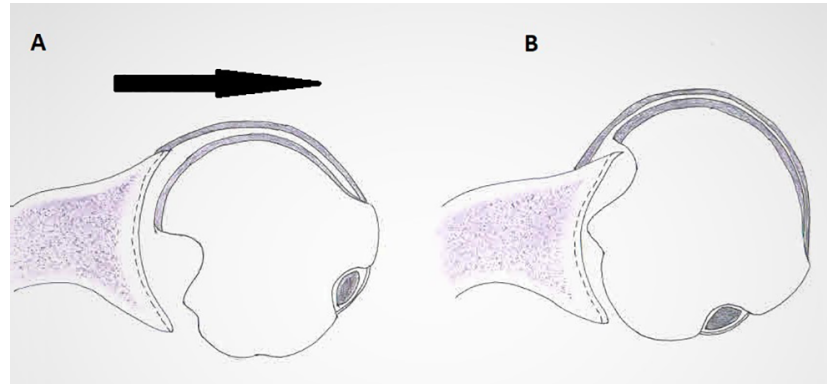

Fig. 4 The articular indentation fracture on the head of the humerus, caused by head impinging over the glenoid rim, the Hill-Sachs lesion.

An ultrasound scan has the added advantage of dynamic assessment and helps to evaluate the rotator cuff and the biceps-labral complex. ${ }^{12}$

There are many classifications for shoulder instability. The most common classification, according to the direction of instability is unidirectional, bidirectional, and multidirectional. In unidirectional instability, 95\% of cases are anterior instability and $5 \%$ posterior. If there is a partial separation of the articular surface, it is subluxation, and dislocation when it is complete. It is divided into acute, subacute, and chronic, according to duration. If it persists for more than 6 weeks, it is a chronic dislocation. It can be due to a macrotrauma, repeated microtrauma, or atraumatic causes.

Rockwood classified it into four types based on the mechanism of instability. Type 1 instability has a history of traumatic subluxation without frank dislocation. Type 2, as in type 1 but with frank dislocation. Types 3 and 4 represent voluntary and atraumatic involuntary subluxation, respectively. The disadvantages of this system include difficulty distinguishing subluxation from dislocation from history. Differentiation of types 3 and 4 will also be difficult. Kessel and Bayley categorized both voluntary and involuntary groups as habitual instability. ${ }^{13}$ Thomas and Matsen put forward a simple classification known as Traumatic Unidirectional Bankart lesion and Surgery (TUBS). These patients have associated lesions of capsuloligamentous complex and Hill-Sachs lesions in the majority of 
patients. ${ }^{14,15}$ The second type is atraumatic, multidirectional, bilateral, rehabilitation is the main treatment, inferior capsular shift, and interval reconstruction in case patients need surgery (AMBRII). ${ }^{16}$ Apart from these two major groups, there are some minor shoulder instabilities also, which include acquired instability, overstressed shoulder (AIOS) and atraumatic minor shoulder instability (AMSI).$^{17}$ The Stanmore system is a comprehensive classification. This places the patient in terms of a set of features into one of the three poles of a triangle. However, it is difficult to categorize them into different poles. Polar type 1, a definite history of trauma, unidirectional instability with Bankart lesion, is like TUBS. Polar type 2 is like AMBRII. Polar type 3 are habitual dislocators, with a significant muscle patterning abnormality. ${ }^{18}$

\section{Discussion}

The natural history of shoulder instability varies in accordance with many factors. Young males with generalized ligamentous laxity have an increased risk of recurrence. Those dislocations associated with a greater tuberosity fracture have less chance of recurrence and greater loss of movements of the shoulder. ${ }^{19}$ In a meta-analysis to determine the natural history of anterior shoulder instability treated nonoperatively, the overall recurrence was $21 \%$, but it was as high as $80 \%$ in boys less than 20 years. ${ }^{20}$ Multiple studies have shown that surgical treatment is beneficial for the treatment of first-time dislocation in young males. ${ }^{21,22}$ No consensus has been reached regarding the position and duration of immobilization in traumatic anterior instability. Shoulder immobilization in the external rotation will help to oppose the torn anteroinferior labrum to the glenoid better than the conventional position of internal rotation. A meta-analysis has shown no additional benefits for sling immobilization longer than 1 week. Patients who are older than 25 years, nonathletes, those without bony lesions, noncompliant patients, and those who have stiff shoulders are the ideal candidates for nonoperative treatment. ${ }^{23,24,25}$

The combined lesions in the glenoid and the humeral head are called bipolar lesions (-Fig. 5). In a bipolar lesion, if the defects are at $90^{\circ}$ humeral abduction and external rotation, the Hill-Sachs lesion engages the glenoid defect, they are on-track lesions. If they are not engaging in $90^{\circ}$ abduction and external rotation, they are off-track lesions (-Fig. 6). ${ }^{26}$ Glenoid defects are located between the 2 and 6 o'clock positions. Hill-Sachs defect is seen in the posterolateral aspect of the humeral head, where it abuts against the anterior glenoid. ${ }^{27}$

When the glenoid defect is less than 25 percent, an arthroscopic or open Bankart repair is indicated. There are comparable results for both arthroscopic and open Bankart repair. ${ }^{28}$ There are reports of a higher rate of failure for arthroscopic repair in young athletes engaged in contact sports. ${ }^{29}$ Considering risk factors such as age $<20$ years, contact sports athletes, level of competitiveness, shoulder hyperlaxity, Hill-Sachs lesion, and loss of contour of glenoid rim, Balg et al devised the instability severity index score. A score of more than 6/10 indicates a high failure rate for Bankart

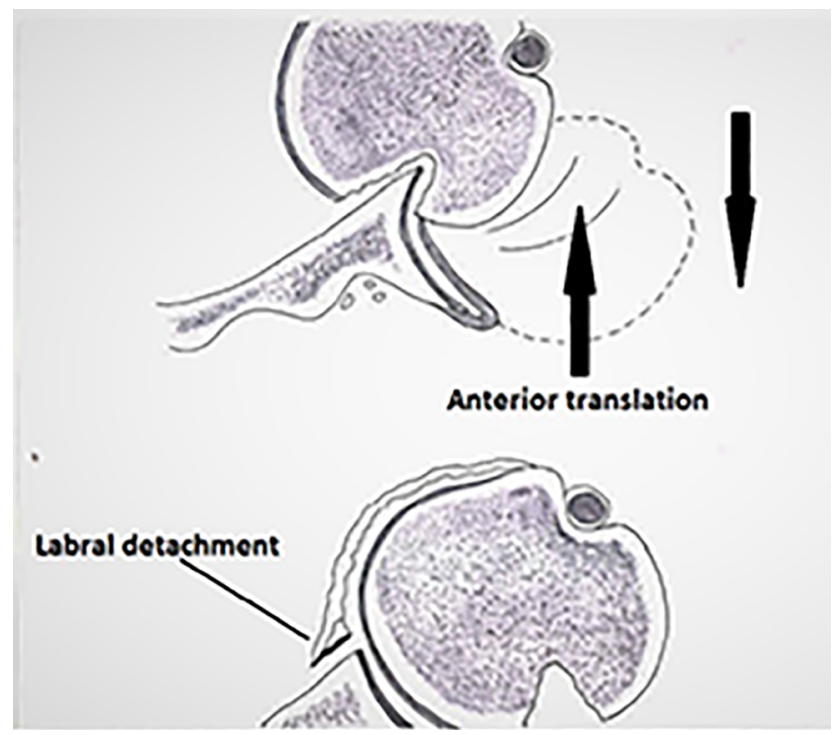

Fig. 5 Bipolar lesion when bone loss occurs in the glenoid and in the humeral head.

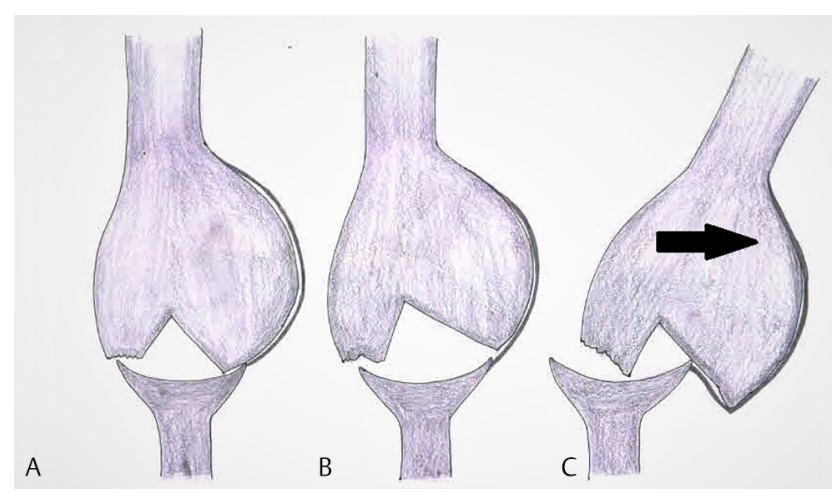

Fig. 6 Engaging and nonengaging type of bipolar lesion.

repair, and such cases require bony reconstruction of the glenoid. In early cases and with large bony Bankart lesions, an open reduction and fixation with screw give good results. ${ }^{19}$ If fixation of the fragment is not possible, then a reconstruction procedure like the Latarjet procedure can be done. In cases where there is more than 25 percentage of glenoid erosion, there is a high failure rate for soft-tissue procedures, and they are better managed with an osseous reconstruction of the glenoid..$^{30,31}$

In a low-demand patient with less than 20 percent humeral head defect, successful management by a short period of immobilization followed by physiotherapy is sufficient. ${ }^{32}$ In high-demand athletes, surgery is indicated. When the defect is between 20 to 40 percent, there are various methods for the management. We can do disimpaction of the humeral head fragment if they present early. Humeroplasty involves elevation of the impacted fragment and supporting it with bone graft or screws. ${ }^{33} \mathrm{~A}$ remplissage procedure can be done for engaging Hill-Sachs lesions. ${ }^{34}$ Weber proximal subcapital humeral osteotomy was used to treat moderate-to-severe Hill-Sachs lesions. This procedure is not done nowadays due to high complications. ${ }^{35}$ There is no consensus in the treatment of a person with more than 40 percent bone loss. 
The treatment in such case is an allograft osseous reconstruction of the humeral head. There are reports of reconstruction of the humeral head, using fresh-frozen humeral head allografts and femoral head allografts ${ }^{36}$ In young patients with good bone quality, partial humeral resurfacing is an emerging technique. ${ }^{37}$ In low-demand or elderly patients, a shoulder hemi/total arthroplasty is an option, depending on the glenoid condition. ${ }^{38}$

Based on the concept of on-track and off-track lesions, DiGiacomo et al proposed an algorithm for the treatment of bipolar lesions. A bipolar lesion with on-track Hill-Sachs can be treated, based on glenoid defect alone. In patients with off-track lesions, both humeral and glenoid defects need to be addressed. ${ }^{26}$ If there is an off-track lesion with less than 25 percent glenoid bone loss, a combined arthroscopic Bankart and remplissage is the procedure of choice. ${ }^{36,39}$

A Bankart repair may be done both by open and arthroscopic routes, with the arthroscopic technique having advantages like smaller incision, shorter operative time, less effect on the range of movements of the shoulder, and lesser blood loss. The main disadvantages are the long learning curve and cost. Results from meta-analyses show that there is a high-failure rate for arthroscopic Bankart repair. ${ }^{40,41}$ Using multiple suture anchors, a proximal shift of anterior capsule, capsular plication, and treating other intra-articular pathologies has helped to increase the success of arthroscopic Bankart repair. ${ }^{42,43}$ In a meta-analysis, it has been found that the rate of dislocation after both open and arthroscopic Bankart repair is almost similar. ${ }^{44}$ There are various reports regarding critical bone loss, which produces dislocation after the arthroscopic procedure, and most of them advocate arthroscopic Bankart repair when bone loss is less than 15 percent. $^{40}$

In 1978, Rowe et al published their long-term results of open Bankart repair, and concluded that it allowed the early return of motion and function with no limitation of shoulder function. ${ }^{45}$ Ogawa et al in an 8-year follow-up of 167 patients reported eight failures, and they suggested that preoperative factors are responsible for the development of postoperative osteoarthritis and the role of surgery is inconclusive. ${ }^{46}$ Many studies have shown good results for an open Bankart repair, although some of them demonstrated radiological shoulder osteoarthritis on follow-up. Newer arthroscopic instrumentation, dedicated residency training, and improved soft-tissue fixation methods have increased the appetite for arthroscopic repair. ${ }^{47-49}$ Clinical results of Bankart repair are similar in both open and arthroscopic methods, but the recurrence rate is twice in contact athletes in the arthroscopic group compared with an open procedure. ${ }^{50}$

In 1954, a thoracic surgeon Michel Letarjet described a reconstruction procedure for shoulder instability. This involved transferring the coracoid process to the anterior glenoid surface and fixing it with a screw. ${ }^{51}$ A similar procedure where the coracoid is transferred to the anterior glenoid and suturing it there was described by Arthur Helfet. He attributed this procedure to his mentor Rowley Bristow. Although simple to perform, there were fixation problems, and the displacement of the coracoid process was a concern.
There was also difficulty in revising the procedure due to altered anatomy on account of the splitting of the subscapularis muscle in the Bristow procedure. Letarjet procedure has got a resurgence in recent years due to the failure of arthroscopic Bankart repair in athletes where critical glenoid defect is more than 13.5 to 15 percent..$^{52}$ The Letarjet procedure produces lesser recurrence and minimum loss of external rotation compared with arthroscopic Bankart repair in borderline glenoid defects. ${ }^{53}$ It is difficult to put two screws parallel to the glenoid flush, with the joint in arthroscopic Letarjet. The other problems with the use of screws for fixing coracoids are screw pullout, breakage, fracture of the bone block, and avulsion. The arthroscopic Letarjet procedure requires a steep learning curve. The combined arthroscopic Letarjet and Bankart repair can be used when the glenoid defect is between 15 to 25 percent. $^{54}$ The open Letarjet procedure involves a similar risk of dislocation in contact athletes compared with noncontact athletes. The Letarjet procedure produces excellent results in previously failed stabilization procedures, with a speedy return to sports in athletes. Osteochondral allograft from the distal tibia and graft from the distal end of the clavicle is used for augmentation of the anterior glenoid, similar to the Letarjet procedure. Their outcomes are comparable. ${ }^{55,56}$ Although the Letarjet procedure is an option in older patients with concomitant glenoid defects and rotator cuff problems, there is a higher rate of graft and hardware-related issues and reoperation. ${ }^{57}$ The contraindications for the Letarjet procedure are recurrent anterior instability in patients above the age of 50 years, elderly patients with an associated massive irreparable rotator cuff tear, first-time dislocation in the elderly without large glenoid rim fracture, voluntary anterior dislocations, uncontrolled epileptic patients, young patients with superior labral tear from anterior to posterior (SLAP) lesions, prosthetic anterior instability, and posttraumatic inferior dislocation. ${ }^{1}$ There are many complications like recurrent instability, infection, hardware failure, fracture and nonunion coracoid process, loss of external rotation, and osteoarthritis of the shoulder..$^{58}$

The Eden-Hybinette procedure is the reconstruction of the anterior glenoid using the iliac crest graft. It has got clinical outcomes similar to Letarjet but has a high recurrence rate and a higher rate of postoperative osteoarthritis. ${ }^{59}$ In young athletes, this procedure should be done before the third episode of dislocation, because it has a higher recurrence if it is done after the fourth episode. ${ }^{60}$

The absolute indication for surgery in the humeral head fracture is displaced humeral head fracture with dislocation and a large Hill-Sachs lesion associated with recurrent dislocation. An engaging humeral head defect involving 20 to 35 percent and a head defect of 10 to 25 percent not centered in the glenoid are relative indications. ${ }^{61}$ Remplissage is a French term meaning to fill in. Remplissage involves filling of Hill-Sachs defect using infraspinatus tendon. The procedure was first described by Wolf et al in 2007. It is used as an adjunct procedure along with the arthroscopic Bankart procedure to address large engaging Hill-Sachs defects. ${ }^{62}$ The remplissage technique consists of transforming an intra-articular humeral head defect into an extra-articular 
lesion, thus reducing the risk of engagement. It consists of posterior capsulodesis and infraspinatus tenodesis into HillSachs lesion. For off-track Hill-Sachs lesion with subcritical glenoid bone loss, satisfactory outcome has been achieved using the Remplissage procedure at par with Letarjet. ${ }^{63}$ For engaging Hill-Sachs lesion, arthroscopic Bankart procedure with remplissage provides better clinical outcomes with no recurrence, even though there is some restriction of external rotation. ${ }^{64}$ The reported complications are low tenosynovitis of the long head of biceps and ulnar nerve palsy. ${ }^{65}$

Weber osteotomy is used in shoulder instability as a salvage procedure in large engaging Hill-Sachs lesion. It is useful for those large Hill-Sachs defects which cannot be managed with a segmental allograft or limited arthroplasty. It involves a derotational osteotomy at the level of the surgical neck by retroverting the humeral head fragment relative to the shaft. There can be a loss of internal rotation and osteoarthritis of the shoulder due to the variability of derotation. ${ }^{35}$

Putti-Platt procedure was a soft-tissue operation in earlier days. It was technically easier than Bankart repair. It involves double breasting of the longitudinally split capsule-subscapularis flap, securing the medial limb to the anterior glenoid and lateral limb over it. It decreases the external rotation. ${ }^{66}$ In a comparative study involving 62 patients over 10 years between Bankart repair and PuttiPlatt assessment of pain and function, there was little difference between both methods ${ }^{67}$ There is an increasing incidence and severity of osteoarthritis, pain, and limitation of external rotation following Putti-Platt procedure. ${ }^{3}$

Posterior instability is seen in $5 \%$ of shoulder instability. It is commonly seen after trauma and less symptomatic than anterior instability. Most of the time diagnosis is missed. ${ }^{68}$ Traumatic posterior instability occurs when the injury occurs when the shoulder is in flexion, adduction, and internal rotated position. Atraumatic instability occurs in collagen diseases and glenoid hypoplasia and retroversion of the glenoid. Most of the patients present with pain and instability. The posterior stress test, jerk test, and load and shift test are used for detection. Plain radiography, CT, and MRI are useful investigations. They can detect glenoid hypoplasia, retroversion, reverse Bankart lesion, reverse Hill-Sachs lesion, reverse humeral avulsion of the glenohumeral ligament, and SLAP tear lesions. Most cases can be managed by nonoperative methods. ${ }^{69}$ Strengthening of dynamic stabilizers like posterior deltoid, external rotators, and periscapular muscles for compensating the lost stability due to static stabilizers is done. Surgery is done in patients where nonoperative treatment fails. It is successful in posttraumatic unidirectional instabilities and both open and arthroscopic procedures are used. In patients with recurrent posttraumatic subluxations, involuntary atraumatic subluxation, voluntary positional posterior subluxation, and open posteroinferior capsular shift procedure give the best results. The indications for arthroscopic procedures are the same. But it is ideal for those with reverse Bankart lesion. Bankart lesions can be repaired using suture anchors, and redundancy of the posterior capsule is reduced by capsular shift or plication procedure. Generalized ligamentous laxity, bony avulsion of glenohumeral ligaments, and failed previous surgeries are contraindications for arthroscopic procedures. ${ }^{70}$ Patients with acquired focal glenoid bone defects can be reconstructed with anatomic intra-articular bone graft to restore the glenoid arc or with an extra-articular bone graft as a buttress to prevent the humeral head subluxation. Open wedge glenoid osteotomy to correct the retroversion is also done. ${ }^{71}$

Multidirectional instability of the shoulder is a poorly defined, not well understood yet common condition. Most patients present in the third or fourth decade with nonspecific activity-related pain. A high-index of suspicion is essential for its diagnosis. Always look for features of generalized ligamentous laxity. Sulcus sign and hyperabduction test are usually positive. Investigations are done similar to other types of instabilities. Rehabilitation is the treatment of choice for patients presenting with multidirectional instability of the shoulder. Evaluation and treatment of scapulothoracic dyskinesia is the main focus of therapy. Surgical treatment is indicated in patients who are symptomatic after nonoperative treatment. It should be individualized to address the anatomic cause of shoulder instability. Many procedures like glenoid osteotomy, labral augmentation, and capsuloligamentous reconstruction exist. Capsuloligamentous techniques, including open inferior capsular shift, thermal capsulorrhaphy, and arthroscopic placation, are commonly used procedures. ${ }^{72,73}$

\section{Conclusion}

Shoulder instability is common in young athletes. Most of the time it got unnoticed in atraumatic and microtraumatic causes. A thorough physical examination and investigations are needed for establishing the diagnosis. Treatment is based on the type of pathological lesions. Arthroscopic and open procedures are available. Both have their indications and advantages. Arthroscopy is preferred as a first-line treatment. Open procedures are useful in complex primary and revision surgeries. Let us hope that newer procedures with low-recurrence rates and failures will come in the future for the treatment of shoulder instability.

\section{Authors' Contributions}

B.Z. conceptualized the idea, helped in collecting data, analysis, statistics, writing, and editing the manuscript. The rest of the authors helped in collecting data, analysis, statistics, writing, and editing the manuscript.

\section{Conflict of Interest}

None declared.

\section{References}

1 Domos P, Lunini E, Walch G. Contraindications and complications of the Latarjet procedure. Shoulder Elbow 2018;10(1):15-24

2 Walton J, Paxinos A, Tzannes A, Callanan M, Hayes K, Murrell GA. The unstable shoulder in the adolescent athlete. Am J Sports Med 2002;30(5):758-767 
3 Kiss J, Mersich I, Perlaky GY, Szollas L. The results of the Putti-Platt operation with particular reference to arthritis, pain, and limitation of external rotation. J Shoulder Elbow Surg 1998;7(5):495-500

4 O'Brien SJ, Neves MC, Arnoczky SP, et al. The anatomy and histology of the inferior glenohumeral ligament complex of the shoulder. Am J Sports Med 1990;18(5):449-456

5 van der Zwaag HM, Brand R, Obermann WR, Rozing PM. Glenohumeral osteoarthrosis after Putti-Platt repair. J Shoulder Elbow Surg 1999;8(3):252-258

6 Owens BD, Nelson BJ, Duffey ML, et al. Pathoanatomy of first-time, traumatic, anterior glenohumeral subluxation events. J Bone Joint Surg Am 2010;92(7):1605-1611

7 Kim DS, Yoon YS, Kwon SM. The spectrum of lesions and clinical results of arthroscopic stabilization of acute anterior shoulder instability. Yonsei Med J 2010;51(3):421-426

8 Grondin P, Leith J. Case series: combined large Hill-Sachs and bony Bankart lesions treated by Latarjet and partial humeral head resurfacing: a report of 2 cases. Can J Surg 2009;52(3):249-254

9 Saccomanno MF, Fodale M, Capasso L, Cazzato G, Milano G. Generalized joint laxity and multidirectional instability of the shoulder. Joints 2014;1(4):171-179

10 Lizzio VA, Meta F, Fidai M, Makhni EC. Clinical evaluation and physical exam findings in patients with anterior shoulder instability. Curr Rev Musculoskelet Med 2017;10(4):434-441

11 Jain NB, Wilcox RB II, Katz JN, Higgins LD. Clinical examination of the rotator cuff. PM R 2013;5(1):45-56

12 Ruiz Santiago F, Martínez A, Tomás Muñoz P, Pozo Sánchez J, Zarza Pérez A. Imaging of shoulder instability. Quant Imaging Med Surg 2017;7(4):422-433

13 Farrar NG, Malal JJ, Fischer J, Waseem M. An overview of shoulder instability and its management. Open Orthop J 2013;7(1):338-346

14 Thomas SC, Matsen FA III. An approach to the repair of avulsion of the glenohumeral ligaments in the management of traumatic anterior glenohumeral instability. J Bone Joint Surg Am 1989;71(4):506-513

15 Varacallo M, Musto MA, Mair SD, Anterior Shoulder Instability. Treasure Island (FL): StatPearls Publishing; 2020

16 Lippitt SB, Harryman DT, Sidles JA, Matsen FA. Diagnosis and management of AMBRI syndrome. Tech Orthop 1991;6(1): 61-74

17 Burkhart SS, Morgan CD, Kibler WB. Shoulder injuries in overhead athletes. The "dead arm" revisited. Clin Sports Med 2000;19(1):125-158

18 Lewis A, Kitamura T, Bayley JIL, II. The classification of shoulder instability: new light through old windows! Curr Orthop 2004;18(2):97-108

19 Dussing F, Plachel F, Grossauer T, et al. Anterior shoulder dislocation and concomitant fracture of the greater tuberosity: clinical and radiological results. Obere Extrem 2018;13 (3):211-217

20 Wasserstein DN, Sheth U, Colbenson K, et al. The true recurrence rate and factors predicting recurrent instability after nonsurgical management of traumatic primary anterior shoulder dislocation: a systematic review. Arthroscopy 2016;32(12):2616-2625

21 Polyzois I, Dattani R, Gupta R, Levy O, Narvani AA. Traumatic first time shoulder dislocation: surgery vs non-operative treatment. Arch Bone Jt Surg 2016;4(2):104-108

22 Hasebroock AW, Brinkman J, Foster L, Bowens JP. Management of primary anterior shoulder dislocations: a narrative review. Sports Med Open 2019;5(1):31

23 Chalidis B, Sachinis N, Dimitriou C, Papadopoulos P, Samoladas E, Pournaras J. Has the management of shoulder dislocation changed over time? Int Orthop 2007;31(3):385-389
24 Paterson WH, Throckmorton TW, Koester M, Azar FM, Kuhn JE. Position and duration of immobilization after primary anterior shoulder dislocation: a systematic review and meta-analysis of the literature. J Bone Joint Surg Am 2010;92(18):2924-2933

25 Itoi E, Hatakeyama Y, Kido T, et al. A new method of immobilization after traumatic anterior dislocation of the shoulder: a preliminary study. J Shoulder Elbow Surg 2003;12(5):413-415

26 Di Giacomo G, Itoi E, Burkhart SS. Evolving concept of bipolar bone loss and the Hill-Sachs lesion: from "engaging/non-engaging" lesion to "on-track/off-track" lesion. Arthroscopy 2014;30(1):90-98

27 Itoi E. 'On-track' and 'off-track' shoulder lesions. EFORT Open Rev 2017;2(8):343-351

28 Rabinowitz J, Friedman R, Eichinger JK. Management of glenoid bone loss with anterior shoulder instability: indications and outcomes. Curr Rev Musculoskelet Med 2017;10(4):452-462

29 Yamamoto $\mathrm{N}$, Kijima $\mathrm{H}$, Nagamoto $\mathrm{H}$, et al. Outcome of Bankart repair in contact versus non-contact athletes. Orthop Traumatol Surg Res 2015;101(4):415-419

30 Balg F, Boileau P. The instability severity index score. A simple pre-operative score to select patients for arthroscopic or open shoulder stabilisation. J Bone Joint Surg Br 2007;89(11):1470-1477

31 Ramhamadany E, Modi CS. Current concepts in the management of recurrent anterior gleno-humeral joint instability with bone loss. World J Orthop 2016;7(6):343-354

32 Maquieira GJ, Espinosa N, Gerber C, Eid K. Non-operative treatment of large anterior glenoid rim fractures after traumatic anterior dislocation of the shoulder. J Bone Joint Surg Br 2007;89(10):1347-1351

33 Stehle J, Wickwire AC, Debski RE, Sekiya JK. A technique to reduce Hill-Sachs lesions after acute anterior dislocation of the shoulder. Tech Shoulder Elbow Surg 2005;6(4):230-235

34 Camp CL, Dahm DL, Krych AJ. Arthroscopic remplissage for engaging Hill-Sachs lesions in patients with anterior shoulder instability. Arthrosc Tech 2015;4(5):e499-e502

35 Brooks-Hill AL, Forster BB, van Wyngaarden C, Hawkins R, Regan WD. Weber osteotomy for large Hill-Sachs Defects: clinical and CT assessments. Clin Orthop Relat Res 2013;471(8):2548-2555

36 Diklic ID, Ganic ZD, Blagojevic ZD, Nho SJ, Romeo AA. Treatment of locked chronic posterior dislocation of the shoulder by reconstruction of the defect in the humeral head with an allograft. J Bone Joint Surg Br 2010;92(1):71-76

37 Raiss P, Aldinger PR, Kasten P, Rickert M, Loew M. Humeral head resurfacing for fixed anterior glenohumeral dislocation. Int Orthop 2009;33(2):451-456

38 Fox JA, Sanchez A, Zajac TJ, Provencher MT. Understanding the Hill-Sachs lesion in its role in patients with recurrent anterior shoulder instability. Curr Rev Musculoskelet Med 2017;10(4):469-479

39 Katthagen JC, Anavian J, Tahal DS, Millett PJ. Arthroscopic remplissage and open Latarjet procedure for the treatment of anterior glenohumeral instability with severe bipolar bone loss. Arthrosc Tech 2016;5(5):e1135-e1141

40 DeFroda S, Bokshan S, Stern E, Sullivan K, Owens BD. Arthroscopic Bankart repair for the management of anterior shoulder instability: indications and outcomes. Curr Rev Musculoskelet Med 2017;10(4):442-451

41 Mohtadi NGH, Bitar IJ, Sasyniuk TM, Hollinshead RM, Harper WP. Arthroscopic versus open repair for traumatic anterior shoulder instability: a meta-analysis. Arthroscopy 2005;21(6):652-658

42 Boileau P, Villalba M, Héry J-Y, Balg F, Ahrens P, Neyton L. Risk factors for recurrence of shoulder instability after arthroscopic Bankart repair. J Bone Joint Surg Am 2006;88(8):1755-1763 
43 Randelli P, Ragone V, Carminati S, Cabitza P. Risk factors for recurrence after Bankart repair a systematic review. Knee Surg Sports Traumatol Arthrosc 2012;20(11):2129-2138

44 Brophy RH, Marx RG. The treatment of traumatic anterior instability of the shoulder: nonoperative and surgical treatment. Arthroscopy 2009;25(3):298-304

45 Rowe CR, Patel D, Southmayd WW. The Bankart procedure: a long-term end-result study. J Bone Joint Surg Am 1978;60(1):1-16

46 Ogawa K, Yoshida A, Matsumoto H, Takeda T. Outcome of the open Bankart procedure for shoulder instability and development of osteoarthritis: a 5- to 20-year follow-up study. Am J Sports Med 2010;38(8):1549-1557

47 Berendes T, Mathijssen N, Verburg H, Kraan G. The open-modified Bankart procedure: long-term follow-up 'a 16-26-year follow-up study' Arch Orthop Trauma Surg 2018;138(5):597-603

48 Chapus V, Rochcongar G, Pineau V, Salle de Chou É, Hulet C. Ten-year follow-up of acute arthroscopic Bankart repair for initial anterior shoulder dislocation in young patients. Orthop Traumatol Surg Res 2015;101(8):889-893

49 Wang L, Liu Y, Su X, Liu S. A meta-analysis of arthroscopic versus open repair for treatment of Bankart lesions in the shoulder. Med Sci Monit 2015;21:3028-3035

50 Freedman KB, Smith AP, Romeo AA. Cole BJ, Bach BR Jr. Open Bankart repair versus arthroscopic repair with transglenoid sutures or bioabsorbable tacks for recurrent anterior instability of the shoulder: a meta-analysis. Am J Sports Med 2004;32(6):1520-1527

51 Saliken D, Boileau P. Arthroscopic Latarjet-learning curve, results, and complications. Ann Joint 2017;2:70-70

52 Reider B. The non-Latarjet. Am J Sports Med 2018;46(5): 1027-1029

53 Jeon YS, Jeong HY, Lee DK, Rhee YG. Borderline glenoid bone defect in anterior shoulder instability: Latarjet procedure versus Bankart repair. Am J Sports Med 2018;46(9):2170-2176

54 Valenti P, Maroun C, Wagner E, Werthel J-D. Arthroscopic Latarjet procedure combined with Bankart repair: a technique using 2 cortical buttons and specific glenoid and coracoid guides. Arthrosc Tech 2018;7(4):e313-e320

55 Paulino Pereira NR, van der Linde JA, Alkaduhimi $\mathrm{H}$, Longo UG, van den Bekerom MPJ. Are collision athletes at a higher risk of re-dislocation after an open Bristow-Latarjet procedure? A systematic review and meta-analysis. Shoulder Elbow 2018;10(2):75-86

56 Hassebrock JD, Starkweather JR, Tokish JM. Arthroscopic technique for bone augmentation with suture button fixation for anterior shoulder instability. Arthrosc Tech 2019;9(1):e97-e102

57 Narayanan SK, Muthusamy N, Pauldhurai VK. Failed shoulder stabilization procedure in the elderly: reverse shoulder replacement is the treatment ultimum. Int J Res Orthop. 2019;5(5):982
58 Domos P, Lunini E, Ascione F, et al. Clinical and radiographic outcomes of open Latarjet procedure in patients aged 40 years or older. J Shoulder Elbow Surg 2019;28(9):e304-e312

59 Longo UG, Loppini M, Rizzello G, Ciuffreda M, Maffulli N, Denaro V. Latarjet, Bristow, and Eden-Hybinette procedures for anterior shoulder dislocation: systematic review and quantitative synthesis of the literature. Arthroscopy 2014;30(9):1184-1211

60 Gebhard F, Draeger M, Steinmann R, Hoellen I, Hartel W. Post-traumatic recurrent shoulder dislocation and the modified Eden-Hybinette procedure - results from the viewpoint of function. Unfallchirurg 1997;100(10):770-775

61 Valencia Mora M, Ruiz-Ibán MÁ, Heredia JD, Ruiz Díaz R, Cuéllar R. Management of humeral defects in anterior shoulder instability. Open Orthop J 2017;11(6):1011-1022

62 Purchase RJ, Wolf EM, Hobgood ER, Pollock ME, Smalley CC. Hill-sachs "remplissage": an arthroscopic solution for the engaging hill-sachs lesion. Arthroscopy 2008;24(6):723-726

63 Yang JS, Mehran N, Mazzocca AD, Pearl ML, Chen VW, Arciero RA. Remplissage versus modified Latarjet for off-track Hill-Sachs lesions with subcritical glenoid bone loss. Am J Sports Med 2018;46(8):1885-1891

64 Miyamoto R, Yamamoto A, Shitara H, et al. Clinical outcome of arthroscopic remplissage as augmentation during arthroscopic Bankart repair for recurrent anterior shoulder instability. Open Orthop J 2017;11(1):1268-1276

65 Rashid MS, Crichton J, Butt U, Akimau PI, Charalambous CP. Arthroscopic "Remplissage" for shoulder instability: a systematic review. Knee Surg Sports Traumatol Arthrosc 2016;24(2):578-584

66 Collins KA, Capito C, Cross M. The use of the Putti-Platt procedure in the treatment of recurrent anterior dislocation. With special reference to the young athlete. Am J Sports Med 1986;14(5):380-382

67 Salomonsson B, Abbaszadegan H, Revay S, Lillkrona U. The Bankart repair versus the Putti-Platt procedure: a randomized study with WOSI score at 10-year follow-up in 62 patients. Acta Orthop 2009;80(3):351-356

68 Hawkins RJ, Koppert G, Johnston G. Recurrent posterior instability (subluxation) of the shoulder. J Bone Joint Surg Am 1984;66(2):169-174

69 Antosh IJ, Tokish JM, Owens BD. Posterior shoulder instability. Sports Health 2016;8(6):520-526

70 Garcia JC, Jr, Maia LR, Fonseca JR, Zabeu JLA, Garcia JPM. Evaluation of arthroscopic treatment of posterior shoulder instability. Acta Ortop Bras 2015;23(3):134-137

71 Millett PJ, Clavert P, Hatch GF II, Warner JJ. Recurrent posterior shoulder instability. J Am Acad Orthop Surg 2006;14(8):464-476

72 Gaskill TR, Taylor DC, Millett PJ. Management of multidirectional instability of the shoulder. J Am Acad Orthop Surg 2011;19(12):758-767

73 Lebar RD, Alexander AH. Multidirectional shoulder instability. Clinical results of inferior capsular shift in an active-duty population. Am J Sports Med 1992;20(2):193-198 